\title{
Pion decay in magnetic fields
}

\author{
Gunnar S. Bali ${ }^{1,2}$, Bastian B. Brandt ${ }^{3}$, Gergely Endrődi ${ }^{3, \star}$, and Benjamin Gläßle ${ }^{1}$ \\ ${ }^{1}$ Institute for Theoretical Physics, Universität Regensburg, D-93040 Regensburg, Germany \\ ${ }^{2}$ Department of Theoretical Physics, Tata Institute of Fundamental Research, Homi Bhabha Road, Mumbai \\ 400005, India \\ ${ }^{3}$ Institute for Theoretical Physics, Goethe Universität Frankfurt, D-60438 Frankfurt am Main, Germany
}

\begin{abstract}
The leptonic decay of the charged pion in the presence of background magnetic fields is investigated using quenched Wilson fermions. It is demonstrated that the magnetic field opens up a new channel for this decay. The magnetic field-dependence of the decay constants for both the ordinary and the new channel is determined. Using these inputs from QCD, we calculate the total decay rate perturbatively.
\end{abstract}

\section{Introduction}

Background magnetic fields are known to impact significantly on the physics of strongly interacting matter - affecting the hadron spectrum, creating an anisotropy in the ground state and influencing the phase structure of QCD. For reviews on this subject and the most recent results on the phase diagram see, e.g., Refs. [1-3]. Notable examples for systems in nature that exhibit strong magnetic fields include off-central heavy-ion collisions, the inner core of magnetized neutron stars and, possibly, the early stage of the evolution of our universe. The magnetic fields in these situations may range up to $B \approx 10^{14-15} \mathrm{~T}$, so that the interaction between quarks and $B$ becomes as strong as the coupling between quarks and gluons. This induces a competition between QCD and QED physics and leads to exciting new phenomena.

In the hadronic, confined phase, the response of strongly interacting matter to the magnetic field $B$ is encoded in the dependence of the properties of hadrons, e.g., masses and decay rates, on $B$, where we assume $\vec{B}=B \vec{e}_{3}$ with $B>0$. For the low-energy behavior of the theory, the lightest hadrons, i.e. pions, are of primary interest. The magnetic field couples to electric charge and thus introduces a splitting between the neutral and charged pions. For the masses $M_{\pi^{0}}$ and $M_{\pi^{ \pm}}$, this splitting is well known and has been studied in various settings [4-13]. For the neutral pion, the decay rate (i.e. the decay constant $f_{\pi^{0}}$ ) has also been determined using different methods [4, 7, 8, 10-12, 14].

Much less is known about how the magnetic field affects the charged pion decay rate. This might be relevant for magnetized neutron stars [15] with pionic degrees of freedom in their core [16]. In this contribution we calculate, for the first time, the decay rate using the weak interaction Lagrangian, in the lowest order of perturbation theory. As we show below, besides the usual decay constant $f_{\pi^{ \pm}}$ (which was discussed for $B>0$ using chiral perturbation theory in Ref. [7]), the decay rate at $B>0$ involves an additional decay constant that we call $f_{\pi^{ \pm}}^{\prime}$. This new decay constant has so far been

${ }^{\star}$ Speaker, e-mail: endrodi@th.physik.uni-frankfurt.de 
ignored in the literature. We perform the perturbative calculation in the so-called lowest Landau-level approximation, which is valid for magnetic fields much larger than the squared mass of the lepton that is produced in the decay. The perturbative treatment is then complemented by a non-perturbative determination of both decay constants and of the pion mass. In particular, we use lattice simulations employing quenched Wilson quarks at $B>0$. Our results give direct access to the magnetic fielddependence of the full decay rate of charged pions into lepton pairs with a muon or an electron.

\section{Pion decay rate and decay constants}

CPT invariance ensures that pions with positive and negative electric charge have the same masses and decay rates. Below we concentrate on the negatively charged pion. The dominant decay channel is that into a lepton pair

$$
\pi^{-}(p) \rightarrow \ell^{-}(k) \bar{v}_{\ell}(q)
$$

where $\ell^{-}$stands for the charged lepton (either electron $\ell=e$ or muon $\ell=\mu$ ). In Eq. (1) we assigned the momenta $p, k$ and $q$ to the pion, the lepton and the antineutrino, respectively. The lepton mass will be denoted as $m_{\ell}$ below. In the effective Lagrangian of the weak interactions, this decay proceeds via a four-fermion vertex. The corresponding amplitude is the matrix element of the interaction between the initial and final states and reads [17]

$$
\mathcal{M}=\frac{G}{\sqrt{2}} \cos \theta_{c} L^{\mu} H_{\mu},
$$

where $G$ is Fermi's constant, $\theta_{c}$ is the Cabibbo angle and $L_{\mu}$ and $H_{\mu}$ are the matrix elements of the leptonic and hadronic contributions to the charged weak current. The leptonic factor reads

$$
L^{\mu}=\bar{u}_{\ell}(k) \gamma^{\mu}\left(1-\gamma^{5}\right) v_{v}(q)
$$

where $u_{\ell}$ and $v_{v}$ are the bispinor solutions of the Dirac equation for the lepton and for the antineutrino, respectively. The hadronic factor is defined by the matrix element

$$
H_{\mu}=\left\langle 0\left|\bar{d} \gamma_{\mu}\left(1-\gamma^{5}\right) u\right| \pi^{-}(p)\right\rangle .
$$

At zero magnetic field, the parity invariance of QCD dictates that the matrix element of the vector part of the weak current vanishes, since it transforms as an axial vector (and there is no axial vector in the system). Thus, $H_{\mu}$ is entirely determined by the axial vector part of the weak current and is proportional to the pion momentum $p_{\mu}$, being the only Lorentz-vector in the problem. The proportionality factor defines the pion decay constant $f_{\pi^{ \pm}}$. Note that there exist subtleties regarding the definition of decay constants of electrically charged hadrons, see, e.g., Ref. [18]. This however goes beyond the tree-level considerations of this presentation.

In the presence of a background magnetic field (in general a Lorentz-tensor $F_{\mu \nu}$ ), further Lorentz structures that are relevant for weak V-A decays can be formed: the vector $F_{\mu \nu} p^{v}$ and the axial vector $-i \epsilon_{\mu \nu \rho \sigma} F^{v \rho} p^{\sigma} / 2$. Specifically, the matrix element of the vector part of the weak current can be nonzero. For a pion at rest, $p^{\mu}=\left(p_{0}, \vec{p}\right)=\left(M_{\pi^{ \pm}}, 0\right)$, and a magnetic field oriented in the positive $z$ direction, $F_{12}=-F_{21}=B$, the matrix element becomes

$$
H_{\mu}=e^{i M_{\pi^{ \pm} t}}\left[f_{\pi^{ \pm}} M_{\pi^{ \pm}} \delta_{\mu 0}+i f_{\pi^{ \pm}}^{\prime} e B M_{\pi^{ \pm}} \delta_{\mu 3}\right],
$$

where we adopt the normalization convention $f_{\pi^{ \pm}} \approx 130 \mathrm{MeV}$ in the vacuum with physical quark masses, and we measure the magnetic field (which has mass dimension two) in units of the elementary 
electric charge $e>0$. Note that while the complex phase of the individual terms in (5) is a matter of convention, their ratio is physical and follows from the transformation properties of (4) under discrete symmetries. In our convention both decay constants are real.

Using the amplitude (2), the decay rate is given by Fermi's golden rule

$$
\Gamma=\int \mathrm{d} \Phi \sum_{\{s\}}|\mathcal{M}|^{2},
$$

involving an integral over the phase space $\Phi$ and a sum over the intrinsic quantum numbers $s$ of the outgoing particles. For $B=0$ the latter involve the spins $s_{\ell}$ and $s_{v}$ of the leptons. To carry out the sum over these, we need the spin sums for the bispinors in (3). For the charged lepton, this reads

$$
\sum_{s_{\ell}} u_{\ell}^{s_{\ell}}(k) \bar{u}_{\ell}^{s_{\ell}}(k)=\not k+m_{\ell}
$$

while for the neutrino the sum gives $q$, neglecting the tiny neutrino masses. A textbook calculation [17] finally results in

$$
\Gamma(B=0)=\frac{\left(G \cos \theta_{c}\right)^{2}}{8 \pi}\left|f_{\pi^{ \pm}}(0)\right|^{2}\left[M_{\pi^{ \pm}}^{2}(0)-m_{\ell}^{2}\right]^{2} \frac{m_{\ell}^{2}}{M_{\pi^{ \pm}}^{3}(0)},
$$

where we indicated that $f_{\pi^{ \pm}}$and $M_{\pi^{ \pm}}$are understood at $B=0$.

\subsection{Decay rate for $B>0$}

For $B>0$ the above calculation is modified considerably. Here we do not describe the details, which will be included in a forthcoming publication [19], but merely summarize the prime points of the calculation. First of all, we need to include the second contribution to $H_{\mu}$ from Eq. (5). In addition, the bispinor solution $u_{\ell}$ is affected, since the Dirac equation for the charged lepton depends on the magnetic field through the electromagnetic vector potential. In particular, the solutions are quantized and correspond to the so-called Landau levels [20] - states with definite angular momentum in the $z$ direction, labeled by the Landau index $n \in \mathbb{Z}_{0}^{+}$.

Each Landau level carries a degeneracy proportional to the flux $e B \cdot L^{2}$ of the magnetic field through the area $L^{2}$ of the system. Moreover, the energies of the states corresponding to the $n$-th Landau level are bounded from below by $\sqrt{m_{\ell}^{2}+2 n e B}$. This allows us to simplify the problem by considering the limit $e B \gg m_{\ell}^{2}$. For such strong magnetic fields, only the states with $n=0$ will contribute, enabling us to restrict ourselves to leptons in the lowest Landau level (LLL) and to neglect levels with $n>0$. The LLL states ${ }^{1}$ are special because they are effectively one-dimensional, allowing only motion aligned with the magnetic field, and also because the spin $s_{\ell}$ is fixed to be antiparallel to the magnetic field for them.

Taking this into account, the equivalent of Eq. (7) reads

$$
\sum_{\mathrm{LLL}} u_{\ell}^{s_{\ell}}(k) \bar{u}_{\ell}^{s_{\ell}}(k)=\left(k_{\|}+m_{\ell}\right) \cdot \frac{1-\sigma^{12}}{2} \cdot \frac{e B \cdot L^{2}}{2 \pi} .
$$

Here, $k_{\|}=k^{0} \gamma^{0}-k^{3} \gamma^{3}$ emerges due to the one-dimensional nature of the LLL states, and the second factor involving the relativistic spin operator $\sigma^{12}=i \gamma^{1} \gamma^{2}$ projects to states with negative spin. Finally, the third factor results from the sum over the LLL-degeneracy. Note that the corresponding spin sum

\footnotetext{
${ }^{1}$ For the role of LLL states in QCD with $B>0$, see Ref. [21].
} 
for the neutrino is not affected by the magnetic field. Using the spin sum (9), we obtain for the decay rate,

$$
\Gamma(B)=e B \frac{\left(G \cos \theta_{c}\right)^{2}}{2 \pi}\left|f_{\pi^{ \pm}}(B)+i f_{\pi^{ \pm}}^{\prime}(B) e B\right|^{2} \frac{m_{\ell}^{2}}{M_{\pi^{ \pm}}(B)} .
$$

Note that we can assume $G \cos \theta_{c}$ to be independent of $B$, since in this context the latter is a low energy scale, being much smaller than the squared mass of the $W$ boson that mediates the weak interaction.

A sensible way to quantify the impact of the magnetic field on $\Gamma$ is to look at the ratio

$$
\frac{\Gamma(B)}{\Gamma(0)}=4 \frac{\left|f_{\pi^{ \pm}}(B)\right|^{2}+\left|f_{\pi^{ \pm}}^{\prime}(B) e B\right|^{2}}{\left|f_{\pi^{ \pm}}(0)\right|^{2}} \cdot\left(1-\frac{m_{\ell}^{2}}{M_{\pi^{ \pm}}^{2}(0)}\right)^{-2} \frac{e B}{M_{\pi^{ \pm}}^{2}(0)} \frac{M_{\pi^{ \pm}}(0)}{M_{\pi^{ \pm}}(B)},
$$

in which the constants $G$ and $\theta_{c}$ cancel. Remember that this result is only valid in the limit $e B \gg m_{\ell}^{2}$.

\section{Lattice simulations}

To calculate the ratio $\Gamma(B) / \Gamma(0)$ of Eq. (11), it remains to determine the non-perturbative parameters $f_{\pi^{ \pm}}(B), f_{\pi^{ \pm}}^{\prime}(B)$ and $M_{\pi^{ \pm}}(B)$ in QCD. To this end we perform lattice simulations using quenched Wilson quarks. We work with the zero-temperature lattice ensembles generated in Ref. [13], with parameters listed in Tab. 1.

Table 1. The parameters of our lattice ensembles: lattice size, inverse gauge coupling $\beta$ and lattice spacing $a$.

\begin{tabular}{c|c|c}
\hline$N_{s}^{3} \times N_{t}$ & $\beta$ & $a$ \\
\hline $12^{3} \times 36$ & 5.845 & $0.124 \mathrm{fm}$ \\
$16^{3} \times 48$ & 6.000 & $0.093 \mathrm{fm}$ \\
$24^{3} \times 72$ & 6.260 & $0.062 \mathrm{fm}$ \\
\hline
\end{tabular}

For this first study, we performed measurements with various different valence quark masses so that the $B=0$ pion mass spans the range $415 \mathrm{MeV} \leq M_{\pi^{ \pm}} \leq 770 \mathrm{MeV}$. The magnetic field is varied in the window $0 \leq e B<1.5 \mathrm{GeV}^{2}$. Note that the magnetic field modifies the critical bare mass parameter for Wilson fermions by large lattice artefacts, as was first pointed out in Ref. [22]. We correct for this by tuning the bare mass parameter along the magnetic field-dependent line of constant physics determined in Ref. [13].

To calculate the pion mass and the decay constants we investigate the matrix elements $H_{0}$ and $\mathrm{H}_{3}$, defined in Eq. (4). To this end, we create a pion state using the smeared pseudoscalar operator $P^{\dagger}=\bar{d} \gamma^{5} u$ at the source, and annihilate it at the sink using either $P$, the local axial vector operator $A=\bar{u} \gamma_{0} \gamma^{5} d$ or the local vector operator $V=\bar{u} \gamma_{3} d$, to obtain the correlators $C_{P P}, C_{A P}$ or $C_{V P}$, respectively. For more details about our measurement strategy, see Ref. [13]. It follows from Eq. (5) that in Euclidean space-time all three correlators decay exponentially as $\exp \left(-M_{\pi^{ \pm}} x_{4}\right)$, where $x_{4}>0$. This is demonstrated in Fig. 1 for $e B=1.3 \mathrm{GeV}^{2}$ and a zero-magnetic field pion mass of $M_{\pi^{ \pm}}=770 \mathrm{MeV}$.

We fit the three correlators simultaneously (for sufficiently large values of $x_{4}$ ) using the functions

$$
C_{O P}(t)=c_{O P}\left[e^{-M_{\pi^{ \pm}} x_{4}} \pm e^{-M_{\pi^{ \pm}}\left(N_{t} a-x_{4}\right)}\right], \quad O=P, A, V,
$$

where the positive sign in front of the second term is taken for $O=P$ and the negative sign for $O=A$ and $O=V$. The decay constants are obtained from the amplitudes of the exponential decays as

$$
f_{\pi^{ \pm}}=Z_{A} \cdot \frac{\sqrt{2} c_{A P}}{\sqrt{M_{\pi^{ \pm}} c_{P P}}}, \quad i f_{\pi^{ \pm}}^{\prime} e B=Z_{V} \cdot \frac{\sqrt{2} c_{V P}}{\sqrt{M_{\pi^{ \pm}} c_{P P}}},
$$




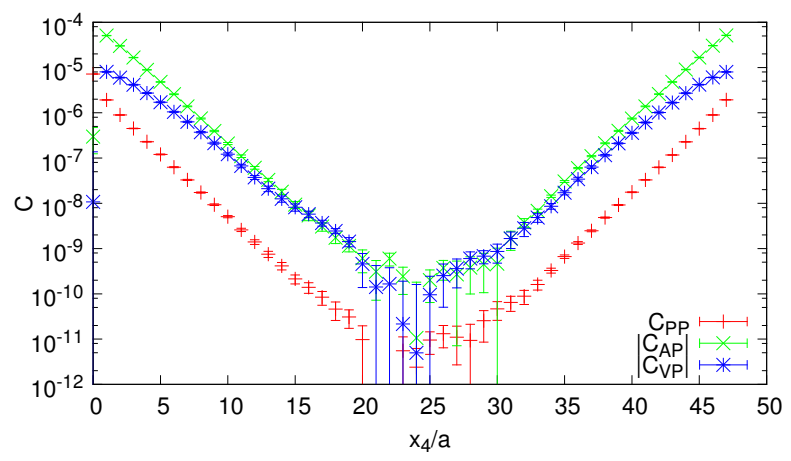

Figure 1. Pseudoscalar-pseudoscalar (red), pseudoscalar-axial vector (green) and pseudoscalar-vector (blue) correlators, measured on our $16^{3} \times 48$ ensemble. Note that $C_{A P}$ and $C_{V P}$ are odd under time reversal and change sign at $x_{4} / a=N_{t} / 2$; the plot shows the modulus of these correlators.

where $Z_{A}$ and $Z_{V}$ are the multiplicative renormalization constants of the axial vector and vector currents, respectively. Up to lattice artefacts, these ultraviolet quantities are expected to be independent of the magnetic field (which is an infrared parameter). We employ the $B=0$ non-perturbative results of Ref. [23] (see also Ref [24]) and fit these in combination with the asymptotic perturbative two-loop results of [25] (see also Ref [26]) to a Padé parametrization.

The results for the pion mass and for the decay constants - in units of $M_{\pi^{ \pm}}(0)$ and of $f_{\pi^{ \pm}}(0)$, respectively - are shown in Fig. 2 for a $B=0$ pion mass of $415 \mathrm{MeV}$. For $M_{\pi^{ \pm}}$, the three lattice spacings lie reasonably close to each other, indicating small discretization errors, at least for $e B<$ $0.8 \mathrm{GeV}^{2}$. (Note that without the magnetic field-dependent tuning of the bare quark mass [13], lattice artefacts would be larger.) For comparison, we also consider the mass of a point-like scalar particle of charge $e, M_{\phi}(B)=\sqrt{M_{\pi^{ \pm}}^{2}(0)+e B}$, which lies quite close to our results for $M_{\pi^{ \pm}}(B)$. This trend has already been observed in the literature, both using dynamical staggered [5], quenched Wilson [6] and quenched overlap quarks [9].

Our results for the decay constants are plotted in the right panel of Fig. 2. $f_{\pi^{ \pm}}$shows no significant dependence on $B$ for the range of magnetic fields that we study here. We also have a clear signal for $f_{\pi^{ \pm}}^{\prime} e B$, showing an initial linear increase, followed by a plateau as $B$ grows. The behavior at low magnetic fields is described by a linear coefficient $f_{\pi^{ \pm}}^{\prime} / f_{\pi^{ \pm}} \approx 0.8 \mathrm{GeV}^{-2}$. In the right panel of Fig. 2 we also include the prediction for $f_{\pi^{ \pm}}$from chiral perturbation theory [7], which suggests a gradual enhancement of $f_{\pi^{ \pm}}(B)$ and is consistent with our results within our present uncertainties of about $10 \%$.

Next we investigate the dependence of the results on the quark mass. We consider three sets of measurements with $B=0$ pion masses of $415 \mathrm{MeV}, 628 \mathrm{MeV}$ and $770 \mathrm{MeV}$. In Fig. 3 we plot the combination $\sqrt{\left|f_{\pi^{ \pm}}(B)\right|^{2}+\left|f_{\pi^{ \pm}}^{\prime}(B) e B\right|^{2}} / f_{\pi^{ \pm}}(0)$ - the square of which characterizes the magnetic fielddependence of the full decay rate, Eq. (11). The plot reveals that to our current accuracy, quark mass effects for our $a=0.093 \mathrm{fm}$ ensemble are invisible in this particular combination. Our results for the other lattice spacings show the same behavior.

Finally, we speculate about the decay rate at the physical point, $M_{\pi^{ \pm}}(0)=138 \mathrm{MeV}$. Assuming that the QCD factor is not significantly modified as the quark mass is reduced further, we take our results for the lowest quark mass and insert these into the formula for the full decay 

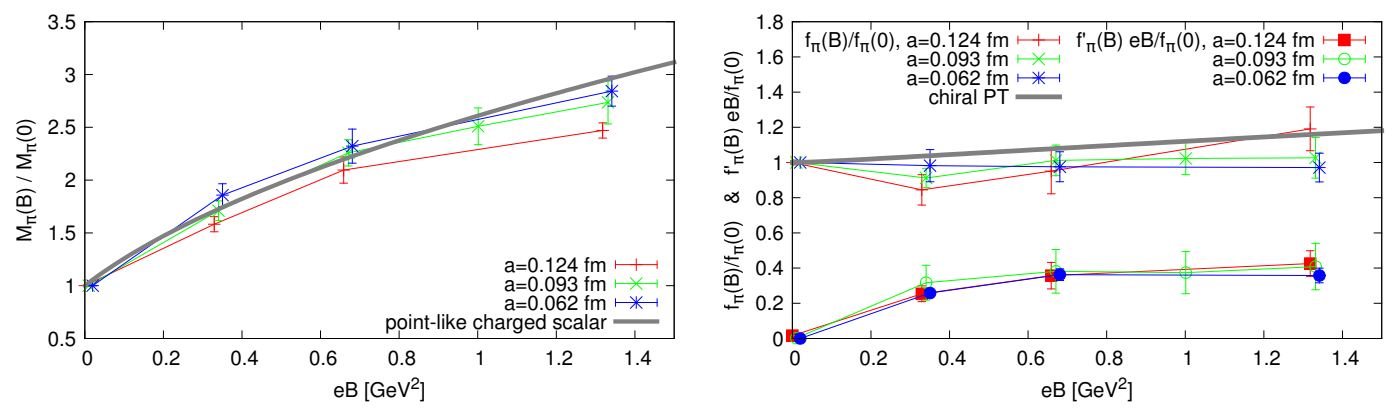

Figure 2. The mass of the charged pion (left panel) and the two decay constants $f_{\pi^{ \pm}}$and $f_{\pi^{ \pm}}^{\prime} e B$ (right panel) as functions of the magnetic field for a $B=0$ pion mass of $415 \mathrm{MeV}$, as measured on three different lattice ensembles. The solid curve in the left panel depicts the mass of a point-like charged scalar particle that interacts only with the magnetic field. The solid curve in the right panel shows the prediction of chiral perturbation theory for $f_{\pi^{ \pm}}(B)$ [7]. The lines connecting the data points merely serve to guide the eye. The points have been shifted horizontally for better visibility.

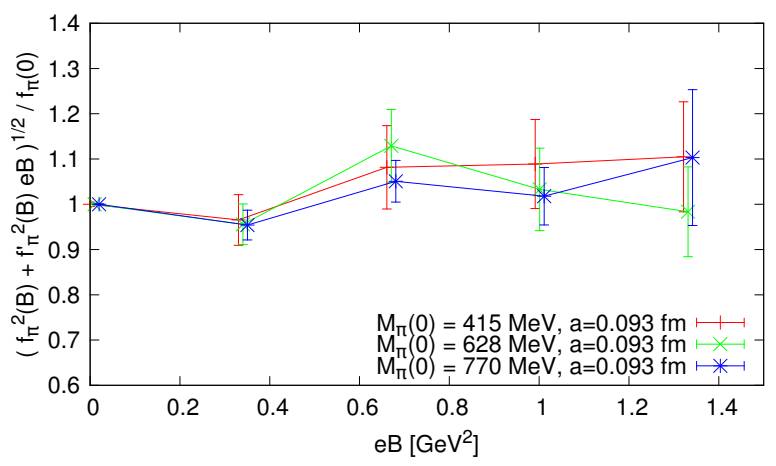

Figure 3. The magnetic field-dependence of the combination $\sqrt{\left|f_{\pi^{ \pm}}(B)\right|^{2}+\left|f_{\pi^{ \pm}}^{\prime}(B) e B\right|^{2}} / f_{\pi^{ \pm}}(0)$, which appears in the decay rate (11) for different $B=0$ pion masses. The points have been shifted horizontally for better visibility.

rate (11), however, replacing the pion mass with $M_{\pi^{ \pm}}(0)=138 \mathrm{MeV}$. We use the free-case formula $M_{\pi^{ \pm}}(B)=\sqrt{M_{\pi^{ \pm}}^{2}(0)+e B}$, which describes our results sufficiently accurately, as we demonstrated in the left panel of Fig. 2. The so-obtained estimate for the decay rate of the charged pion at the physical point is shown in Fig. 4. In the left panel we consider the decay into electrons $\ell=e$, whereas the right panel depicts the results for the decay into muons $\ell=\mu$. The only difference between the two cases is the lepton mass $m_{e}=0.5 \mathrm{MeV}$ versus $m_{\mu}=105 \mathrm{MeV}$. Remember that since we employed the lowest Landau-level approximation, the decay rate formula is only valid for magnetic fields well above the respective squared lepton masses, which is the case for all our data points.

The results reveal a fast approach towards the continuum limit. For magnetic fields of the order $e B \approx 0.3 \mathrm{GeV}^{2}$ - which is a typical value relevant for the physical situations discussed in the introduction - a drastic enhancement of $\Gamma$ is clearly visible. For the electronic decay the rate enhances by 

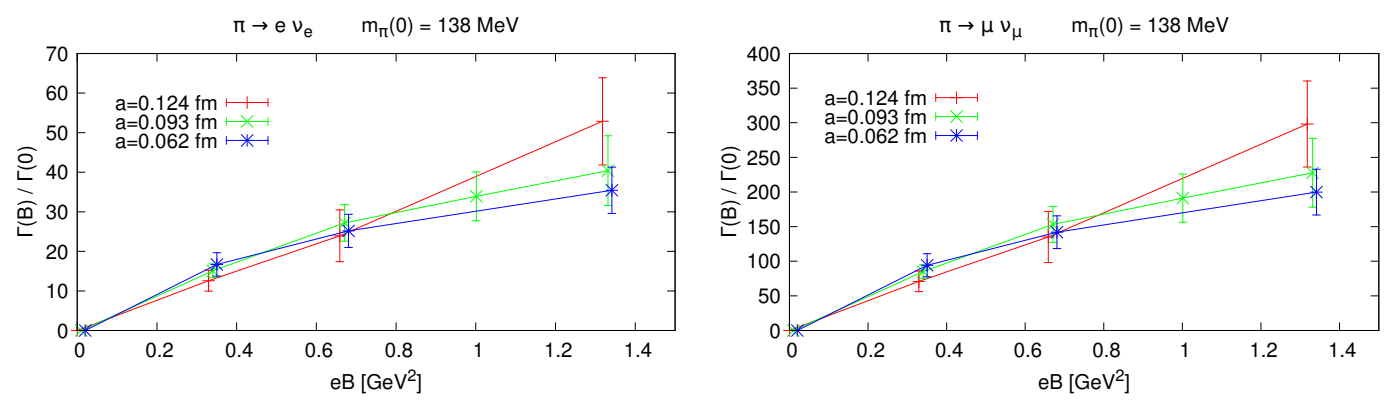

Figure 4. The decay rate of the process $\pi^{-} \rightarrow e^{-} \bar{v}_{e}$ (left panel) and for $\pi^{-} \rightarrow \mu^{-} \bar{v}_{\mu}$ (right panel) in units of the respective $B=0$ values. Here we took the physical pion mass in the formula (11) and assumed - as Fig. 3 suggests - that the QCD factor does not change considerably between the physical point and $M_{\pi^{ \pm}}=415 \mathrm{MeV}$. The points have been shifted horizontally for better visibility.

a factor $\sim 20$ with respect to $\Gamma(0)$, while for the muonic decay the enhancement is by more than two orders of magnitude. Thus, the average lifetime of the charged pion is reduced drastically.

We point out that the ratio of the electronic and the muonic decay rates is independent of the magnetic field,

$$
e B \gg m_{\mu}^{2}: \quad \frac{\Gamma\left(\pi \rightarrow e \bar{v}_{e}\right)}{\Gamma\left(\pi \rightarrow \mu \bar{v}_{\mu}\right)}=\frac{m_{e}^{2}}{m_{\mu}^{2}} \approx 2.27 \cdot 10^{-5},
$$

and is suppressed by a factor of five compared to the $B=0$ ratio [27]. Therefore, for strong background magnetic fields the muonic decay becomes even more dominant than it already is at $B=0$.

\section{Conclusions}

In this contribution we studied the mass and the decay rate of charged pions in the presence of background magnetic fields. To determine the rate of decay into leptons, we have performed a tree-level perturbative calculation in the electroweak theory, assuming that the magnetic field is well above the squared mass of the charged lepton, $e B \gg m_{\ell}^{2}$. This assumption enables the use of the lowest Landaulevel approximation for the outgoing lepton state, which simplifies the calculation considerably.

The perturbative calculation involves the matrix element of the weak current between the vacuum and a pion state. We have demonstrated for the first time, that for nonzero background fields this matrix element is characterized by two independent decay constants $f_{\pi^{ \pm}}$and $f_{\pi^{ \pm}}^{\prime}$. Both decay constants were determined non-perturbatively on the lattice using quenched Wilson fermions. We investigated the dependence of the results on the lattice spacing and on the quark mass (or, equivalently, on the $B=0$ pion mass), revealing modest discretization errors and quark-mass effects.

We find the muonic partial decay rate, which amounts to over $99.9 \%$ of the total decay rate, to be enhanced by two orders of magnitude for magnetic fields of around $e B \approx 0.3 \mathrm{GeV}^{2}$, that are of phenomenological relevance. We predict a similar enhancement by a factor of around 20 for the electronic decay rate. The ratio (14) of the decay rates in the two channels is found to be independent of $B$, and of all non-perturbative quantities - thus this prediction applies irrespective of the results of our lattice simulations. Our findings may have applications for the physics of magnetars. 
Acknowledgments This research was funded by the DFG (Emmy Noether Programme EN 1064/2-1 and SFB/TRR 55). BB acknowledges support from the Frankfurter Förderverein für Physikalische Grundlagenforschung. We thank Zoltán Fodor and Andreas Schäfer for illuminating discussions.

\section{References}

[1] D.E. Kharzeev, K. Landsteiner, A. Schmitt, H.U. Yee, Lect. Notes Phys. 871, 1 (2013), 1211.6245

[2] J.O. Andersen, W.R. Naylor, A. Tranberg, Rev. Mod. Phys. 88, 025001 (2016), 1411.7176

[3] G. Endrődi, JHEP 07, 173 (2015), 1504.08280

[4] N.O. Agasian, I. Shushpanov, JHEP 0110, 006 (2001), hep-ph/0107128

[5] G. Bali, F. Bruckmann, G. Endrődi, Z. Fodor, S. Katz et al., JHEP 1202, 044 (2012), 1111.4956

[6] Y. Hidaka, A. Yamamoto, Phys. Rev. D87, 094502 (2013), 1209.0007

[7] J.O. Andersen, JHEP 1210, 005 (2012), 1205.6978

[8] S. Fayazbakhsh, S. Sadeghian, N. Sadooghi, Phys. Rev. D86, 085042 (2012), 1206.6051

[9] E.V. Luschevskaya, O.E. Solovjeva, O.A. Kochetkov, O.V. Teryaev, Nucl. Phys. B898, 627 (2015), 1411.4284

[10] S.S. Avancini, R.L.S. Farias, M. Benghi Pinto, W.R. Tavares, V.S. Timteo, Phys. Lett. B767, 247 (2017), 1606.05754

[11] R. Zhang, W.j. Fu, Y.x. Liu, Eur. Phys. J. C76, 307 (2016), 1604.08888

[12] S. Mao, Y. Wang (2017), 1702.04868

[13] G.S. Bali, B.B. Brandt, G. Endrődi, B. Gläßle (2017), 1707.05600

[14] S. Fayazbakhsh, N. Sadooghi, Phys. Rev. D88, 065030 (2013), 1306. 2098

[15] R.C. Duncan, C. Thompson, Astrophys. J. 392, L9 (1992)

[16] A.B. Migdal, E. Saperstein, M. Troitsky, D. Voskresensky, Phys.Rept. 192, 179 (1990)

[17] L. Okun, Leptons and Quarks, North-Holland Personal Library (Elsevier Science, 2013), ISBN 9780444596215

[18] A. Patella, PoS LATTICE2016, 020 (2017), 1702 . 03857

[19] B.B. Brandt, G. Bali, G. Endrődi, B. Gläßle, in preparation.

[20] L. Landau, E. Lifshits, Quantum Mechanics: Non-relativistic Theory, Butterworth-Heinemann (Butterworth-Heinemann, 1977), ISBN 9780750635394

[21] F. Bruckmann, G. Endrődi, M. Giordano, S.D. Katz, T.G. Kovács, F. Pittler, J. Wellnhofer (2017), 1705.10210

[22] B.B. Brandt, G. Bali, G. Endrődi, B. Gläßle, PoS LATTICE2015, 265 (2016), 1510. 03899

[23] V. Gimenez, L. Giusti, F. Rapuano, M. Talevi, Nucl. Phys. B531, 429 (1998), hep-lat/9806006

[24] M. Göckeler, R. Horsley, H. Oelrich, H. Perlt, D. Petters, P.E.L. Rakow, A. Schäfer, G. Schierholz, A. Schiller, Nucl. Phys. B544, 699 (1999), hep-lat/9807044

[25] A. Skouroupathis, H. Panagopoulos, Phys. Rev. D79, 094508 (2009), 0811.4264

[26] G.S. Bali, F. Bursa, L. Castagnini, S. Collins, L. Del Debbio, B. Lucini, M. Panero, JHEP 06, 071 (2013), 1304.4437

[27] K.A. Olive et al. (Particle Data Group), Chin. Phys. C38, 090001 (2014) 